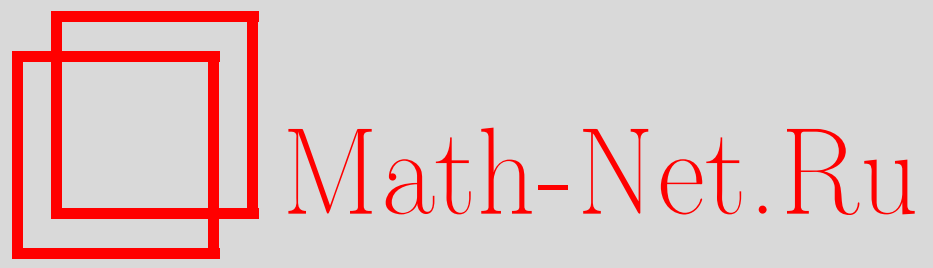

Обцероссийский математический портал

А. Н. Печень, Об одном асимптотическом разложении в квантовой теории, Матем. заметки, 2004, том 75, выпуск 3, 459-461

DOI: https://doi.org/10.4213/mzm551

Использование Общероссийского математического портала Math-Net.Ru подразумевает, что вы прочитали и согласны с пользовательским соглашением http://www.mathnet.ru/rus/agreement

Параметры загрузки:

IP : 54.224 .135 .184

26 апреля 2023 г., 11:07:23 


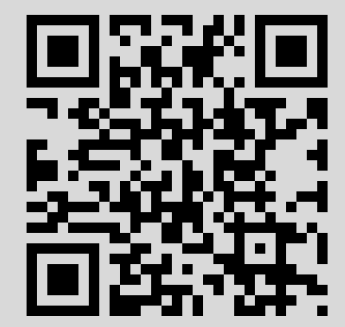




\section{ОБ ОДНОМ АСИМПТОТИЧЕСКОМ РАЗЛОЖЕНИИ В КВАНТОВОЙ ТЕОРИИ}

\section{А. Н. Печень}

Асимптотические методы [1] широко применяются в квантовой механике, квантовой теории поля, статистической физике. В данной заметке строится асимптотическое разложение, которое возникает при изучении квантовых открытых систем, а именно, при изучении динамики таких систем на больших временах в режиме слабой связи. Известно, что динамика квантовой системы, взаимодействующей с резервуаром, на больших временах (в стохастическом пределе) описывается уравнением Шрёдингера с квантовым белым шумом [2], квантовыми стохастическими и мастер-уравнениями [3], [4]. Важной задачей является изучение поправок к стохастическому пределу. Как было установлено в [5], эти поправки описываются уравнениями, в которые входят операторы рождения и уничтожения, действующие в псевдогильбертовых пространствах, т.е. в пространствах с индефинитной метрикой (см. [6], [7]). Эти операторы были названы квантовым мультипольным иумом.

В [5] было явно построено представление квантового дипольного шума, введенного в [8], в пространстве Фока с индефинитной метрикой. В данной заметке впервые строится представление алгебры всего набора квантовых мультипольных шумов в бесконечном тензорном произведении гильбертовых и псевдогильбертовых пространств и с исполззованием этого представления формулируется основная теорема об асимптотическом разложении свободной эволюции операторов рождения и уничтожения (после перерастяжки времени) по операторам мультипольного шума. Это разложение понимается в смысле асимптотического разложения корреляционных функций. Такое асимптотическое разложение (нулевым членом которого является квантовый белый шум) возникает и играет важную роль при изучении временной динамики квантовых открытых систем на болшшх временах в случае слабого взаимодействия с резервуаром.

1. Некоторые определения. Пусть $S(\mathbb{R})$ обозначает пространство Шварца быстро убывающих комплекснозначных функций на прямой; $C^{\infty}\left(\mathbb{R}^{d}\right)$ - пространство комплекснозначных бесконечно дифференцируемых функций на $\mathbb{R}^{d}, C_{0}^{\infty}\left(\mathbb{R}^{d}\right)$ - пространство комплекснозначных бесконечно дифференцируемых функций с компактным носителем на $\mathbb{R}^{d}$.

Квантовым мультипольньм шумом называется *-алгебра с образуюшими $\left\{c_{n}^{ \pm}(f) \mid n \in \mathbb{N} \cup\{0\}\right.$, $f \in S(\mathbb{R})\}$, удовлетворяюшими коммутационным соотношениям

$$
\left[c_{m}^{-}(f), c_{n}^{+}(h)\right]=\delta_{n, m} i^{n} \gamma_{n} \int_{\mathbb{R}} \overline{f^{(n)}(t)} h(t) d t, \quad\left[c_{m}^{-}(f), c_{n}^{-}(h)\right]=\left[c_{m}^{+}(f), c_{n}^{+}(h)\right]=0
$$

Работа выполнена при финансовой поддержке Российского фонда фундаментальных исследований, грант № 02-01-01084, INTAS YSF 01/1-200 и NATO-CNR Fellowship.

(C) А. Н. ПЕчень 
где $\delta_{n, m}$ - символ Кронекера, $\gamma_{n}$ - вещественные числа, $\gamma_{0}>0, f^{(n)}$ обозначает $n$-ю производную функции $f, \bar{f}$ - функция, комплексно сопряженная к $f$. Предполагается, что $\gamma_{n} \neq 0$. Мы будем использовать обозначения $c^{+}(f)=\int d t f(t) c^{+}(t), c^{-}(f)=\int d t \bar{f}(t) c^{-}(t)$, где $c_{n}^{ \pm}(t)$ - операторно-значные обобщенные функции такие, что

$$
\left[c_{n}^{-}(t), c_{m}^{+}(\tau)\right]=\delta_{n, m} i^{n} \gamma_{n} \delta^{(n)}(\tau-t)
$$

и $\delta^{(n)}(\tau-t)$ обозначает $n$-ю производную $\delta$-функции Дирака.

Псевдогильбертовым пространством называется пара $(\mathscr{H}, \hat{\eta})$, где $\mathscr{H}$ - гильбертово пространство, а $\hat{\eta}: \mathscr{H} \rightarrow \mathscr{H}$ - линейный ограниченный самосопряженный оператор такой, что $\hat{\eta}^{2}=1$ (оператор с такими свойствами является унитарным). Оператор $\hat{\eta}$ называется метрическим опеpатором. Он определяет индефинитное скалярное произведение $\langle\cdot, \cdot\rangle$ равенством $\langle f, h\rangle:=$ $(f, \hat{\eta} h)$, где $f, h \in \mathscr{H}$ и $(\cdot, \cdot)$ - положительно определенное скалярное произведение в $\mathscr{H}$.

2. Представление квантового мультипольного шума. В данном пункте построено представление квантового $2^{n}$-польного шума $c_{n}(f)$ с $\gamma_{n}=1$ (обобщение на случай произвольных $\gamma_{n}$ производится непосредственно) операторами рождения и уничтожения в симметричном пространстве Фока (см. [7])

$$
\mathscr{F}_{n}=\Gamma\left(\mathscr{H}_{n}\right)=\bigoplus_{k=0}^{\infty} \mathscr{H}_{n}^{\otimes_{\mathrm{sym}}^{k}}
$$

над одночастичным (гильбертовым для четных $n$ и псевдогильбертовым для нечетных $n$ ) пространством $\mathscr{H}_{n}$. Это одночастичное (псевдо) гильбертово пространство строится как пополнение пространства Шварца $S(\mathbb{R})$ по норме, индуцированной скалярным произведением

$$
(f, h)_{\mathscr{H}_{n}}=\frac{1}{2 \pi} \int|x|^{n} \overline{\tilde{f}(x)} \tilde{h}(x) d x
$$

где $\tilde{f}(x) \equiv(F f)(x)=\int e^{i t x} f(t) d t$ - преобразование Фурье функции $f$. Метрический оператор $\eta_{n}$ на $\mathscr{H}_{n}$ определим как единственное расширение из плотного подпространства $S(\mathbb{R}) \subset \mathscr{H}_{n}$ на все $\mathscr{H}_{n}$ линейного оператора $\eta=F^{-1} \operatorname{sign}(x) F$. Здесь $\operatorname{sign}(x)=1$, если $x \geqslant 0$, и -1 иначе. Очевидно, что оператор $\eta_{n}$ удовлетворяет свойствам оператора метрики: $\eta_{n}=\eta_{n}^{+}$и $\eta_{n}^{2}=1$. Индефинитное скалярное произведение в $\mathscr{H}_{n}$ для нечетных $n$ имеет вид $\langle f, h\rangle_{\mathscr{H}_{n}}=\left(f, \eta_{n} h\right)_{\mathscr{H}_{n}}$. Для четных $n$ положим по определению $\langle f, h\rangle_{\mathscr{H}_{n}} \equiv(f, h)_{\mathscr{H}_{n}}$, так что в этом случае это положительно определенное скалярное произведение. В общем случае можно написать $\langle f, h\rangle_{\mathscr{H}_{n}} \equiv\left(f, \eta_{n}^{n} h\right)_{\mathscr{H}_{n}}$.

Индефинитная (для нечетных $n$ ) метрика в $\mathscr{H}_{n}$ индуцирует индефинитную метрику в $\mathscr{F}_{n}$ посредством оператора $\hat{\eta}_{n}=\Gamma\left(\eta_{n}^{n}\right)$ (вторичное квантование унитарного оператора $\eta_{n}$ ), которую мы обозначим как $\langle\cdot, \cdot\rangle_{\mathscr{F}_{n}}$.

Пусть $\phi \in \mathscr{F}_{n}$ - финитный вектор $\phi=\left(f_{0}, f_{1}\left(t_{1}\right), \ldots, f_{k}\left(t_{1}, \ldots, t_{k}\right), \ldots\right)$, т.е. такой, что $f_{k}=$ $0, k \geqslant N$ для некоторого $N \in \mathbb{N}$. Операторы квантового $2^{n}$-польного шума $c_{n}^{+}(f)$ и $c_{n}^{-}(f)(f \in$ $S(\mathbb{R}))$ определены на плотном в $\mathscr{F} n$ множестве финитных векторов и действуют на $k$-частичную компоненту финитного вектора $\phi$ как

$$
\begin{aligned}
& \left(c_{n}^{+}(f) f_{k}\right)_{k+1}\left(t_{1}, \ldots, t_{k+1}\right)=\frac{1}{\sqrt{k+1}} \sum_{i=1}^{k+1} f\left(t_{i}\right) f_{k}\left(t_{1}, \ldots, \hat{t}_{i}, \ldots, t_{k+1}\right), \\
& \left(c_{n}^{-}(f) f_{k}\right)_{k-1}\left(t_{1}, \ldots, t_{k-1}\right)=i^{n} \sqrt{k} \int \overline{f^{(n)}(t)} f_{k}\left(t, t_{1}, \ldots, t_{k-1}\right) d t
\end{aligned}
$$

В (3) $\hat{t}_{i}$ означает, что аргумент $t_{i}$ опущен.

ПРЕДЛОЖЕнИЕ 1. Операторы $c_{n}^{ \pm}(f)$, заданные в (псевдо) зильбертовом пространстве $\left(\mathscr{F}_{n}, \hat{\eta}_{n}\right)$ формулами (3), (4), удовлетворяют на финитных векторах каноническим коммутачионным соотношениям

$$
\left[c_{n}^{-}(f), c_{n}^{+}(h)\right]=\langle f, h\rangle_{\mathscr{H}_{n}}=i^{n} \int_{\mathbb{R}} \overline{f^{(n)}(t)} h(t) d t
$$


и соотношению

$$
\left\langle c_{n}^{-}(f) \phi, \psi\right\rangle_{\mathscr{F}_{n}}=\left\langle\phi, c_{n}^{+}(f) \psi\right\rangle_{\mathscr{F}_{n}},
$$

которое означает, что оператор уничтохсения $c_{n}^{-}(f)$ (псевдо) әрмитово сопряхен к оператору рохсдения $c_{n}^{+}(f)$.

Пусть Г есть бесконечное тензорное произведение гильбертовых пространств $\mathscr{F} n$ (по фон Нейману [9]):

$$
\Gamma=\bigotimes_{n=0}^{\infty} \mathscr{F}_{n}
$$

со стабилизирующей последовательностью $\left\{\phi_{n}\right\}$, где $\phi_{n}$ - вакуумный вектор в $\mathscr{F} n$. Мы отождествляем операторы $c_{n}^{ \pm}(f)$ в $\mathscr{F}_{n}$ с операторами, действующими в $\Gamma$ как $c_{n}^{ \pm}(f)$ на $n$-ю компоненту тензорного произведения и как 1 на остальных сомножителях. Метрический оператор в Г определим как $\hat{\eta}=1 \otimes \hat{\eta}_{1} \otimes 1 \otimes \hat{\eta}_{3} \otimes \ldots$. В результате мы построили представление квантового мультипольного шума в псевдогильбертовом пространстве $(\Gamma, \hat{\eta})$.

3. Теорема о разложении свободной эволюции операторов рождения и уничтожения. Пусть

$$
\Gamma\left(L^{2}\left(\mathbb{R}^{d}\right)\right)=\bigoplus_{n=0}^{\infty} L^{2}\left(\mathbb{R}^{d}\right)^{\otimes_{\text {sym }}^{n}}
$$

обозначает симметричное (бозонное) пространство Фока с вакуумным вектором $\Omega$ и скалярным произведением $(\cdot, \cdot)$.

Для $f, g \in C_{0}^{\infty}\left(\mathbb{R}^{d}\right)$ определены операторы рождения и уничтожения $a^{ \pm}(f)$, заданные на плотном в $\Gamma\left(L^{2}\left(\mathbb{R}^{d}\right)\right)$ множестве финитных векторов и удовлетворяюшие на этом множестве каноническим коммутационным соотношениям

$$
\left[a^{-}(g), a^{+}(f)\right]=(g, f)_{L^{2}\left(\mathbb{R}^{d}\right)}, \quad\left(a^{+}(f) \phi, \psi\right)=\left(\phi, a^{-}(f) \psi\right) .
$$

Пусть $\omega: \mathbb{R}^{d} \rightarrow \mathbb{R}$ - бесконечно дифференцируемая ограниченная снизу функция и $S_{t}, t \in \mathbb{R},-$ однопараметрическая унитарная группа в $L^{2}\left(\mathbb{R}^{d}\right)$, действующая на $f \in L^{2}\left(\mathbb{R}^{d}\right)$ как $\left(S_{t} f\right)(p)=$ $e^{i t \omega} f(p)$. В физических приложениях функция играет роль закона дисперсии. Типичным примером является $\omega(p)=p^{2} / 2 m$ для некоторого $m>0$. При изучении временной динамики квантовой двухуровневой системы, взаимодействующей с резервуаром, появляется сдвиг в законе дисперсии на шширину перехода между энергетическими уровнями системы $\omega_{0}$, так что закон дисперсии принимает вид $\omega(p)=p^{2} / 2 m-\omega_{0}$.

Обозначим

$$
A_{\lambda}^{ \pm}(t)=\frac{1}{\lambda} a^{ \pm}\left(S_{t / \lambda^{2}} g\right), \quad \Phi_{0}=\phi_{0} \otimes \phi_{1} \otimes \cdots \otimes \phi_{n} \otimes \cdots \in \Gamma .
$$

Tеорема 1. Пусть $g \in C_{0}^{\infty}\left(\mathbb{R}^{d}\right), \omega \in C^{\infty}\left(\mathbb{R}^{d}\right)$, supp $g \cap\left\{k_{0} \mid \nabla \omega\left(k_{0}\right)=0\right\}=\varnothing$. Тогдa для произвольных натуральных $n$ и имеет место равенство следующих корреляторов (как обобщенных функиий из $S^{\prime}\left(\mathbb{R}^{n}\right)$ по переменным $\left.t_{1} \ldots t_{n}\right)$ :

$$
\left(\Omega, A_{\lambda}^{\epsilon_{1}}\left(t_{1}\right) \ldots A_{\lambda}^{\epsilon_{n}}\left(t_{n}\right) \Omega\right)=\left\langle\Phi_{0}, \sum_{i_{1}=0}^{N} \lambda^{i_{1}} c_{i_{1}}^{\epsilon_{1}}\left(t_{1}\right) \cdots \sum_{i_{n}=0}^{N} \lambda^{i_{n}} c_{i_{n}}^{\epsilon_{n}}\left(t_{n}\right) \Phi_{0}\right\rangle+o\left(\lambda^{N}\right),
$$

әде $\epsilon_{i}= \pm$, в правой части $\langle\cdot, \cdot\rangle$ обозначает индефинитное скалярное произведение в Г и квантовые операторы мультипольного шума $c_{n}^{ \pm}(t)$ (операторно-значные обобщенные функи,ии) удовлетворяют коммутаиионным соотношениям (2) с

$$
\gamma_{n}=\frac{i^{n}}{n !} \int_{-\infty}^{\infty} \sigma^{n} d \sigma \int_{\mathbb{R}^{d}} e^{i \sigma \omega(k)}|g(k)|^{2} d k
$$


ЗАмЕч АниЕ 1. Утверждение теоремы можно интерпретировать как следующее асимптотическое операторное разложение:

$$
a^{ \pm}\left(S_{t / \lambda^{2}} g\right) \sim \sum_{n=0}^{\infty} \lambda^{n+1} c_{n}^{ \pm}(t), \quad \lambda \rightarrow 0 .
$$

Доказательство теоремы основано на использовании теоремы Вика (см. [10]), принципа локальности (см. [11]) и полученного с его помощью асимптотического разложения

$$
\frac{1}{\lambda^{2}} \int d k|g(k)|^{2} e^{i t \omega(k)(t-\tau) / \lambda^{2}} \sim \sum_{n=0}^{\infty}(i \lambda)^{n} \gamma_{n} \delta^{(n)}(t-\tau) .
$$

\section{СПИСОК ЦИТИРОВАННОЙ ЛИТЕРАТУРЫ}

1. Маслов В. П. Асимптотические методы и теория возмущений. М.: Наука, 1988. 2. Accardi L., Lu Y. G., Volovich I. V. Quantum Theory and its Stochastic Limit. Berlin: Springer, 2002. 3. Чеботарев А. M. Lectures on Quantum Probability. Mexico: SMM, 2000. 4. Холево А. С. (ред.) Квантовые случайные процессы и открытые системы: Сб. статей 1982-1982 гг. М.: Мир, 1988. 5. Pechen A. N., Volovich I. V. // Infinite-Dimensoinal Analysis, Quantum Probability and Related Topics. 2002. V. 5. Р. 441-464. 6. Азизов Т. Я., Й охвидов И. С. Основы теории линейных операторов в пространствах с индефинитной метрикой. М.: Наука, 1986. 7. Боголюбов Н.Н., Логунов А. А., Оксак А. И., Тодоров И. Т. Общие принципы квантовой теории поля. М.: Наука, 1987. 8. Волович И. В. Quantum Dynamics and Black Noise. Доклад на международной конференции по квантовой информации, Meijo University, 1998. 9. von Neumann J. // Composito Math. 1938. V. 6. Р. 1. 10. Боголюбов Н. Н., Ширков Д. В. Квантовые поля. М.: Наука, 1980. 11. Федорюк М. В. Метод перевала. М.: Наука, 1977. 